\title{
REVIEW
}

\section{British Andrology Society guidelines for the assessment of post vasectomy semen samples (2002)}

\author{
P Hancock, E McLaughlin, for the British Andrology Society
}

J Clin Pathol 2002;55:812-816

The British Andrology Society guidelines for the assessment of post vasectomy semen samples recommend that initial assessment is undertaken 16 weeks post vasectomy and after the patient has produced at least 24 ejaculates. The laboratory should examine a freshly produced seminal fluid specimen by direct microscopy and if no sperm are seen the centrifugate should be examined for the presence of motile and non-motile spermatozoa. It is recommended that the clinician should give clearance after the production of two consecutive sperm free ejaculates. In cases of persistent identification of non-motile spermatozoa the referring clinician should advise the patient regarding the cessation of other contraceptive precautions. Surgeons are responsible both preoperatively and postoperatively for the counselling of couples regarding complications and the possibility of late recanalisation after clearance.

See end of article for authors' affiliations

...................

Mr P Hancock, Department of Microbiology, Yeovil District Hospital, Higher

Kingston, Yeovil, Somerset BA2 1 4ÁT, UK;

hancp@est.nhs.uk

Accepted for publication 26 June 2002

\begin{abstract}
A s a learned society, the British Andrology Society (BAS) has sought to improve standards of diagnostic andrology services within the UK via educational courses and the publication of guidelines for the screening of semen donors. ${ }^{1}$ In 1993, the BAS conducted a pilot study within the membership and was instrumental in the setting up of the UK National External Quality Assurance Scheme (UKNEQAS) for Andrology. ${ }^{2}$ In April 1999, at the fifth meeting of the UKNEQAS for Andrology user group at Manchester Royal Infirmary Postgraduate Medical Centre, the UKNEQAS specialist advisory group debated with the delegates the problems associated with the assessment of semen samples after vasectomy. Over 100 delegates were present and took part in discussion sessions, and it was proposed from the floor and endorsed by all present that the BAS should produce guidelines for laboratories undertaking post vasectomy semen analysis, in an attempt to promote best practice in this difficult but medico legally important area of laboratory andrology practice. ${ }^{3}$
\end{abstract}

\section{AIMS}

The aims of the BAS guidelines on the assessment of semen samples after vasectomy are to give guidance to laboratory staff to ensure standardisation of seminal analysis protocols and reporting of results. After consultation with members of the
British Association of Urological Surgeons, the Royal College of Obstetricians and Gynaecologists (RCOG), and the Family Planning Association (FPA), the committee and members of BAS have produced these guidelines. The BAS guidelines do not deal with the counselling of patients or discuss the indications for male sterilisation, all of which are considered in the RCOG guidelines on male and female sterilisation. ${ }^{4}$

\section{INTRODUCTION}

Vasectomy is regarded as one of the safest and most effective methods of birth control, ${ }^{5}$ and it is popular particularly among the many stable couples who have completed their families. ${ }^{6} \mathrm{Al}-$ though vasectomy is a relatively simple procedure, it is not without complications, ${ }^{7}$ and careful preoperative counselling is required. ${ }^{8}$ Failure to provide and to document adequate preoperative information and counselling are common causes of litigation. ${ }^{39}$ Complications include the short term problems of wound infection, scrotal haematoma formation, and primary surgical failure, together with the longer term issues of chronic epididymal pain ${ }^{10}$ and spontaneous reversal. ${ }^{71} 12$

\section{FERTILITY OF RESIDUAL SPERM POST SURGERY}

Evidence suggests that virtually all ejaculates will contain potentially fertile spermatozoa immediately after vasectomy, ${ }^{13}$ and that patients should continue with contraceptive precautions until they have been advised by their clinician otherwise. In the small number of studies performed so far, the spermatozoa remaining within the male reproductive tract rapidly become immotile, often within a few days of the vasectomy operation, ${ }^{13-15}$ and usually by three weeks. ${ }^{16}$

Although standardised follow up studies are scarce, ${ }^{17}$ it has been suggested that several unplanned pregnancies have occurred immediately after vasectomy, and these have been attributed not to technical failure of surgery or recanalisation, but to the patient failing to comply with instructions regarding continuing contraceptive precautions. ${ }^{10}$

\section{EARLY FAILURE OF SURGERY}

Both technical failure (such as missed vasa deferentia) and early recanalisation of the vas deferens have been documented. ${ }^{18}$ In a large study of

Abbreviations: BAS, The British Andrology Society; FPA, Family Planning Association; RCOG, Royal College of Obstetricians and Gynaecologists; UKNEQAS, UK

National External Quality Assurance Scheme 
16796 vasectomy operations carried out within one centre in the UK, the surgeons reported a failure to achieve sterility in 72 men. This early failure rate was quoted as $0.36 \%,{ }^{19}$ and has been linked to operator experience ${ }^{819}$ and the technique used by the surgeon. ${ }^{30}$ Early failures were usually identified by the presence of motile spermatozoa in ejaculates examined three to six months postoperatively, ${ }^{18}{ }^{19}$ and surveys have suggested that many surgeons will opt to repeat surgery ${ }^{721}$ as soon as possible.

\section{LATE FAILURE}

Late failure is defined as the presence of spermatozoa in ejaculates after documented azoospermia in two post vasectomy semen analyses. ${ }^{12}{ }^{22}$ Although uncommon, the late failure rate has been reported to be $0.04-0.08 \%{ }^{18}{ }^{19}$ and recanalisation is usually only discovered after pregnancy in the female partner. ${ }^{12} 1822{ }^{23}$ It has been suggested that because women may not inform their partners of a pregnancy for fear of being accused of infidelity, but may seek a termination, the late failure rate may be an underestimate. ${ }^{10}$ Similarly, the identification of spermatozoa in $9.7 \%$ of ejaculates from a group of 186 men undergoing semen assessment prereversal ${ }^{24}$ of their vasectomy also suggests an under reporting of late recanalisation.

\section{LABORATORY ASSESSMENT OF POST VASECTOMY SEMEN SAMPLES \\ Sample collection}

The RCOG has recommended that before vasectomy ${ }^{4}$ the patient should have received information from his clinician regarding the likelihood of a successful vasectomy operation and the possibility of recanalisation. It is recommended that such information should be given both verbally and in writing and repeated by the clinician at the time of final clearance. ${ }^{34}$

The laboratory should ensure that the clinician has clear written instructions for sample collection (appendix 1) and suitable specimen containers to give to the patient. Laboratories should advise clinicians that men must be asked to collect their entire ejaculate by masturbation into the non-toxic sample container provided. ${ }^{25}$

Semen samples should be collected after an abstinence period of no less than 48 hours and no more than seven days, and maintained at body temperature before delivery to the laboratory (ideally within one hour of production in line with current World Health Organisation protocols ${ }^{25}$ ). It is recommended that laboratories endeavour to examine samples within four hours of production. If this is not feasible, the period elapsing between production and examination should be stated on the report form. However, in cases of the persistent presence of non-motile sperm in the ejaculate, further fresh samples should be examined. These samples must be delivered to the laboratory within one hour of production and again maintained at or near, but not exceeding, body temperature until receipt in the laboratory. It is suggested that an appointment be made with the laboratory to ensure that the sample is examined immediately on arrival, to establish sperm motility. Postal delivery of samples is not recommended because sperm motility declines rapidly with time. ${ }^{25}$

\section{"It is recommended that laboratories endeavour to examine samples within four hours of production"}

If, however, there is a significant risk of patient noncompliance or hardship, laboratories may make exceptions and accept samples by post. Laboratories accepting postal samples should ensure that patients understand the sample/ request form labelling requirements and the current regulations for the transport and packaging of samples (see HSE 1996). Postal samples must abide by these requirements and be sent by first class post.
In cases of paternity dispute, samples must be delivered personally to the laboratory and all details confirmed with the patient before the sample is examined.

Many men will produce ejaculates that contain residual (usually non-motile) spermatozoa for some weeks after surgery. ${ }^{26}$ The BAS recommends that patients should be instructed to ensure that they have had at least 24 ejaculations, ${ }^{5618}$ and preferably wait at least 16 weeks before submitting a first semen sample for examination..$^{27-29}$ This will reduce the number of false positive samples and thus minimise both patient inconvenience and repeat laboratory assessment. $^{28}$

On delivery of the semen sample to the laboratory reception, staff should ensure that the collection container is clearly labelled with at least three patient identifiers (such as name, date of birth, and clinic number) and the corresponding form is completed in full with details of sample collection and abstinence period (appendix 2). If an unlabelled sample container is delivered, for medicolegal reasons the laboratory should not examine the specimen and should request a repeat ejaculate for assessment.

Patients should be given a further collection container and instructed to produce a second ejaculate for examination at an interval of two to four weeks after the initial assessment. In the event that samples delivered by post contain non-motile spermatozoa, it is mandatory that a freshly produced sample be examined within one hour of production to exclude the possibility that motile sperm are present.

\section{Sample examination}

After registration, freshly produced semen samples should be examined as soon as liquefaction is complete, ideally within one hour of production, ${ }^{25}$ and preferably within four hours of production. Before examination, semen samples should be stored either at room temperature or in an incubator at $37^{\circ} \mathrm{C}$. The specimen should not be subjected to extremes of temperature $\left(<20\right.$ or $\left.>40^{\circ} \mathrm{C}\right)$ because this will significantly impair sperm motility.

The sample should be mixed well and a $10 \mu \mathrm{l}$ aliquot pipetted on to a clean non-toxic glass slide using a direct displacement pipette and the semen covered with a $22 \times 22 \mathrm{~mm}$ coverslip. This gives a suitable depth $(20 \mu \mathrm{m})$ to allow any motile sperm present to swim freely. ${ }^{25}$

Using phase contrast microscopy, it is recommended that the entire $(22 \times 2 \mathrm{~mm})$ coverslip area should be examined in a systematic grid search pattern for the presence of sperm. Any sperm detected should be categorised as either motile or nonmotile and reported to the referring clinician (appendix 3).

If no sperm are observed by direct microscopy, the entire semen sample should be transferred using a sterile pipette into an appropriate conical bottomed non-toxic polypropylene centrifuge tube and centrifuged at no less than $3000 \times g$ for 15 minutes. ${ }^{25}$ Failure to centrifuge the sample at the appropriate " $g$ " force may result in the failure to recover spermatozoa from the ejaculate. ${ }^{3031}$

The entire centifugate or "pellet" should be resuspended in a minimum volume of autologous seminal plasma $(<100 \mu \mathrm{l})$ and the entire sample examined systematically (as described above) for the presence of motile and non-motile spermatozoa. ${ }^{25}$ The BAS recommends that an estimation of sperm concentration or numbers of spermatozoa observed in each high power field $(\times 400$ magnification $)$ should be reported to the clinician.

After centrifugation, the pellet may contain a large number of cells including germ cells, epithelial cells, and leucocytes. ${ }^{25}$ The presence of many cells may make the detection of any sperm in the pellet very difficult and the laboratory should advise the clinician that these cells might have obscured any sperm present (appendix 3). Early examination, before the 16 week postoperative period, may exacerbate this problem because of the cellular response. 
In a small minority of cases, when the semen sample is extremely viscous, laboratories may be unable to obtain a suitable centrifuged pellet to examine. In this situation, it is recommended that a repeat sample should be requested. In cases of persistent seminal viscosity, the entire ejaculate may be incubated for 30-60 minutes at room temperature with a protease such as $1 \%$ bromelin (Bromelain, CN Biosciences UK, Beeston, UK). A stock solution of $10 \%$ bromelin in phosphate buffered saline ( $\mathrm{pH}$ 7.2) should be prepared and aliquots stored at $-20^{\circ} \mathrm{C}$ until required. ${ }^{32}$ Aliquots can be thawed and diluted to $1 \%$ with phosphate buffered saline and brought to temperature when required. Incubation with bromelin may immobilise any motile sperm present and the laboratory should advise the clinician accordingly (appendix 3 ).

\section{CLEARANCE}

The RCOG and FPA guidelines state that at least two azoospermic consecutive samples two to four weeks apart must be obtained before contraceptive precautions can be dispensed with. Recent studies have re-emphasised this period as appropriate. ${ }^{29}$ It is the responsibility of clinicians not the laboratory to communicate the results of semen assessments to their patients. ${ }^{4}$ A large proportion of patients (up to 25\%) fail to submit post vasectomy semen specimens for assessment, ${ }^{27} 2833$ or fail to comply with instructions regarding sample collection, ${ }^{25}$ making reliable laboratory assessment impossible. ${ }^{17}$ Proscriptive sample collection may increase the risk of non-compliance by patients; however, this should be minimised by appropriate counselling and laboratory staff should endeavour to ensure patient compliance by accommodating clinical and patient wishes (such as postal delivery of semen specimens) whenever possible.

\section{SPECIAL CLEARANCE}

Persistent non-motile spermatozoa in the initial two ejaculates is not uncommon, with studies reporting up to 33\% nonazoospermic samples at three months, ${ }^{34}$ and $10 \%$ of ejaculates containing non-motile sperm at six months. ${ }^{28}$ In one prospective study, the median time to azoospermia was 10 weeks, with most men (93\%) being azoospermic by week $20 .{ }^{26}$ Similarly, sperm can reappear in ejaculates 12 months after surgery, despite previous sperm free ejaculates. ${ }^{35}$ Over 1000 men taking part in a prospective long term follow up study have submitted semen samples at one, two, and three years post vasectomy. ${ }^{12}$ Twenty men had a positive semen analysis but there were no reports of unwanted pregnancies in the partners of these men. ${ }^{12}$ Discussion in the literature has suggested that the risk of pregnancy occurring from these non-motile sperm is small, ${ }^{27}$ and probably no more than the risk of pregnancy after two azoospermic semen samples, as a result of spontaneous recanalisation. ${ }^{12}{ }^{21}$ It has been suggested that repeat ejaculates seven months after surgery are probably unnecessary because pregnancy is very unlikely to occur. ${ }^{27} 36$

\section{"It is the responsibility of clinicians not the laboratory to communicate the results of semen assessments to their patients"}

Samples in which non-motile sperm are repeatedly detected should be examined within the recommended time periods to optimise motility detection. Before the issuing of "special clearance" the clinician may ask the laboratory to confirm the vital status of the non-motile spermatozoa using an appropriate staining technique. ${ }^{25}$

If any motile sperm or substantial numbers of immotile spermatozoa $\left(>10^{5}\right)$ are detected then the laboratory should inform the clinician promptly because many surgeons will opt to repeat surgery ${ }^{72}$ as soon as possible.

\section{Take home messages}

- This article describes the British Andrology Society (BAS) guidelines for laboratories undertaking post vasectomy semen analysis, which are designed to promote best practice in this difficult but medico legally important area of laboratory andrology practice

- These guidelines recommend that initial assessment is undertaken 16 weeks after vasectomy and after the patient has produced at least 24 ejaculates

- A freshly produced seminal fluid specimen should be examined by direct microscopy and if no sperm are seen the centrifugate should be examined for the presence of motile and non-motile spermatozoa

- Clinicians should give clearance after the production of two consecutive sperm free ejaculates

- When non-motile spermatozoa are persistently identified, the referring clinician should advise the patient on the cessation of contraception

- Surgeons are responsible for the counselling of couples regarding complications and the possibility of late recanalisation after clearance

These men with low numbers of persistent non-motile spermatozoa in their ejaculates (that is, after seven months and at least 24 ejaculations) may be given "special clearance" by their clinician to discontinue other contraceptive precautions following appropriate oral counselling and written advice (appendix 4) regarding the risk of pregnancy. ${ }^{21} 2936$

The BAS recognises the problems associated with patient non-compliance in the production and assessment of post vasectomy samples, as highlighted by various workers. ${ }^{37} 38$ Because single, early ( $<16$ weeks) samples are more likely to give inconclusive results requiring further patient samples, these laboratory guidelines coupled with careful pre vasectomy counselling are designed to ensure that appropriate clearance should be available to men and their surgeons within 20 weeks of the operation. The BAS suggests that close adherence to these guidelines will provide a high quality accurate laboratory service to patients and clinicians following vasectomy.

\section{REVISION OF GUIDELINES}

It is the intention of the BAS committee that these guidelines will be reviewed on a regular basis and updated as necessary.

\section{ACKNOWLEDGEMENTS}

The BAS thanks Dr H Cooling, Senior Clinical Medical Officer in Family Planning, United Bristol Hospitals Trust; Professor J Guillebaud, Medical Director, Margaret Pyke Family Planning Centre London; Mr C Gingell, Consultant Urological Surgeon, North Bristol NHS Trust Department of Urology; Mr T Hargreave Consultant Urological Surgeon, Western General Hospital NHS Trust Edinburgh; Mr C Parker, Consultant Urological Surgeon, East Somerset NHS Trust; Mr J Pryor, Consultant Uroandrologist, Lister Hospital London; Dr C Smith and Dr J Walsh, Clinical Effectiveness Unit, Faculty of Family Planning and Reproductive Health Care, London; and Dr P Trotter, Lead Clinician in Contraception and Sexual Health Family Planning Clinic Taunton and Somerset Hospital for their help and advice.

\section{Authors' affiliations}

P Hancock, Department of Microbiology, Yeovil District Hospital, Higher Kingston, Yeovil, Somerset BA2 1 4AT, UK

E McLaughlin, University of Newcastle, School of Environmental and Life Science, University Drive, Callaghan, New South Wales 2308, Australia 
Appendix 1 Semen analysis (post vasectomy)

Please read these instructions thoroughly

Semen analysis is carried out only by appointment with the laboratory. Appointments can be made by phoning 01234 56789, between 0900 and 1600 hours Monday to Friday. Your first appointment must be at least 16 weeks and 24 ejaculations after your operation.

\section{Collection of the sample}

The specimen must be collected in the morning. You must not have had sex or masturbated for 48 hours before collecting the sample but should have had an abstinence period of no longer than seven days. First wash your hands and genitals with soap and water, please ensure complete removal of all soap residues and dry thoroughly.

\section{The container}

Please use the sterile container provided. Do not open the container until you are ready to produce the sample.

Collect the sample by masturbation, collecting the entire specimen directly into the container. (Do not use a sheath/condom for collection, as they are harmful to sperm. Please note: No other forms of sample collection are acceptable.)

Seal the container immediately after sample collection with the lid; make sure that the lid is on tightly. Do not use adhesive tape. Write your name, date of birth plus date and time of production on the container label. Record your period of abstinence and the date of your operation on the form provided and ensure the name of the requesting consultant/general practitioner plus your name, address, and date of birth is also stated on the form.

\section{Delivery of the sample}

Deliver the sample and completed form to the Pathology Laboratory within one hour of collection. The sample must be examined before it is four hours old, for this reason postal delivery is not acceptable.

\section{** UNLABELLED SPECIMEN POTS WILL NOT BE PROCESSED **}

If you have any difficulties associated with the collection and delivery of your sample please contact the laboratory on telephone number 0123456789.

\section{Appendix 2 Pathology laboratory request form}

Surname

Forename

Date Of Birth

Address

Clinic No.

Requesting Doctor

Date of Vasectomy

\section{Specimen details}

When was the semen sample collected? Date Time a.m./p.m.

Was the whole sample collected? YES/NO

When was the last time you ejaculated before this? Date I_ Time_a.m./p.m.

\section{Appendix 3 Pathology laboratory report form}

Name
Clinic No.
Date sample_t
Delay to examination
Volume hrs
Specimen collection: Complete/Incomplete
Direct microscopy: No sperm seen/__ Motile sperm
seen/_Non-motile sperm seen
Centrifugate: No sperm seen/_ Motile sperm seen/
Vital staining: Live sperm seen/No live sperm seen
Comments:
Centrifugate contained many cells_these may have obscured
any sperm present
Viscous sample treated with bromelin_this may have caused
immotility of sperm present
No further specimens necessary
Repeat sample requested
Clinician to review

\section{Appendix 4 Example letter to patient}

Mr Smith

Miss Jane Jones Consultant Urologist

12 The Avenue, Newtown

15th September 2000

Dear Mr Smith

This letter is to confirm that there were no active sperm present in your post vasectomy semen samples; however, a very small number of non-motile (inactive) sperm were still present in your last sample. There is no evidence that a pregnancy has occurred in men producing these low numbers of inactive sperm.

At your initial appointment we discussed that vasectomy operations are not $100 \%$ successful and there is always a small failure rate of between one in two to three thousand cases. This means that there is a very low but not zero chance that you might conceive in the future.

If at any time in the future you have any problems regarding your vasectomy operation or there is a possibility that Mrs Smith has become pregnant, then you must contact you general practitioner immediately.

Yours sincerely

Miss Jane Jones 


\section{REFERENCES}

1 British Andrology Society. British Andrology Society guidelines for the screening of semen donors for donor insemination (1999). Hum Reprod $1999: 14: 1823-6$

2 Matson PL. External quality assessment for semen analysis and sperm antibody detection: results of a pilot scheme. Hum Reprod 1995; 10:620-5.

3 Preston JM. Vasectomy: common medicolegal pitfalls. BJU Int 2000;86:339-43.

4 RCOG. Male and female sterilisation. London: Royal College of Obstetricians and Gynaecologists, 1999

5 Rajifer J, Bennett CJ. Vasectomy. Urol Clin North Am 1988;15:631-4.

6 Schmidt SS. Vasectomy. Urol Clin North Am 1987;14:149-54.

7 Hendry WF. Vasectomy and vasectomy reversal. Br J Urol 1994;73:337-44.

8 Greek G. Vasectomy. A safe, effective, economical means of sterilization [In process citation]. Postgrad Med 2000;108:173-6;179.

9 Babayan RK, Krane RJ. Vasectomy: what are community standards? Urology 1986;27:328-30.

10 Schwingl PJ, Guess HA. Safety and effectiveness of vasectomy. Fertil Steril 2000;73:923-36

11 Pugh RC, Hanley HG. Spontaneous recanalisation of the divided vas deferens. Br J Urol 1969:41:340-7.

12 Haldar N, Cranston D, Turner E, et al. How reliable is a vasectomy? Long-term follow-up of vasectomised men [letter]. Lancet 2000;356:43-4.

13 Richardson DW, Aitken RJ, Loudon NB. The functional competence of human spermatozoa recovered after vasectomy. J Reprod Fertil 1984:70:575-9

14 Jouannet P, David G. Evolution of the properties of semen immediately following vasectomy. Fertil Steril 1978;29:435-41.

15 Lewis EL, Brazil CK, Overstreet JW. Human sperm function in the ejaculate following vasectomy. Fertil Steril 1984;42:895-8.

16 Edwards IS. Earlier testing after vasectomy, based on the absence of motile sperm. Fertil Steril 1993;59:431-6.

17 Rees RW. Vasectomy: problems of follow up. Proc $R$ Soc Med 1973;66:52-4

18 Alderman PM. The lurking sperm. A review of failures in 8879 vasectomies performed by one physician. JAMA 1988:259:3142-4.

19 Philp T, Guillebaud J, Budd D. Complications of vasectomy: review of 16,000 patients. Br J Urol 1984;56:745-8

20 Verhulst AP, Hoekstra JW. Paternity after bilateral vasectomy. BJU Int 1999;83:280-2.

21 Benger JR, Swami SK, Gingell JC. Persistent spermatozoa after vasectomy: a survey of British urologists. Br J Urol 1995;76:376-9.
22 Philp T, Guillebaud J, Budd D. Late failure of vasectomy after two documented analyses showing azoospermic semen. BM 1984;289:77-9.

23 Smith JC, Cranston D, O'Brien T, et al. Fatherhood without apparent spermatozoa after vasectomy [see comments]. Lancet 1994;344:30.

24 Lemack GE, Goldstein M. Presence of sperm in the pre-vasectomy reversal semen analysis: incidence and implications. J Urol 1996;155:167-9.

25 WHO. Laboratory manual for the examination of human semen and sperm cervical mucus interaction, 4th ed. Cambridge: Cambridge University Press, UK, 1999

26 Cortes M, Flick A, Barone MA, et al. Results of a pilot study of the time to azoospermia after vasectomy in Mexico City. Contraception 1997;56:215-22.

27 Arango Toro O, Andolz Peitivi P, Llado Carbonell C, et al. Post-vasectomy semen in 313 males. Statistical analysis, medical aspects, legal implications. Arch Esp Urol 1993;46:29-34.

28 Smith AG, Crooks J, Singh NP, et al. Is the timing of post-vasectomy seminal analysis important [see comments]? Br J Urol 1998;81:458-60.

29 Gingell C, Crosby D, Carroll R. Review of the complications and medicolegal implications of vasectomy. Postgrad Med J 2001:77:656-9.

30 Lindsay KS, Floyd I, Swan R. Classification of azoospermic samples [letter]. Lancet 1995;345: 1642.

31 Jaffe TM, Kim ED, Hoekstra TH, et al. Sperm pellet analysis: a technique to detect the presence of sperm in men considered to have azoospermia by routine semen analysis. J Urol 1998;159:1548-50.

32 Shulman S, Hu C. A study of the detection of sperm antibody in cervical mucus with a modified immunobead method. Fertil Steril 1992;58:387-91.

33 Badrakumar C, Gogoi NK, Sundaram SK. Semen analysis after vasectomy: when and how many? BJU Int 2000;86:479-81.

34 De Kniiff DW, Vrijhof HJ, Arends J, et al. Persistence or reappearance of nonmotile sperm after vasectomy: does it have clinical consequences? Fertil Steril 1997:67:332-5.

35 O'Brien TS, Cranston D, Ashwin P, et al. Temporary reappearance of sperm 12 months after vasectomy clearance. Br J Urol 1995;76:371-2.

36 Davies AH, Sharp RJ, Cranston D, et al. The long-term outcome following "special clearance" after vasectomy. Br J Urol 1990;66:211-12

37 Bradshaw HD, Rosario DJ, James M, et al. Review of current practice to establish success after vasectomy. Br J Surg 2001;88:290-3.

38 Sivardeen KAZ, Budhoo M. Post vasectomy analysis: call for a uniform evidence-based protocol. Ann R Coll Surg Engl 2001;83:177-9.

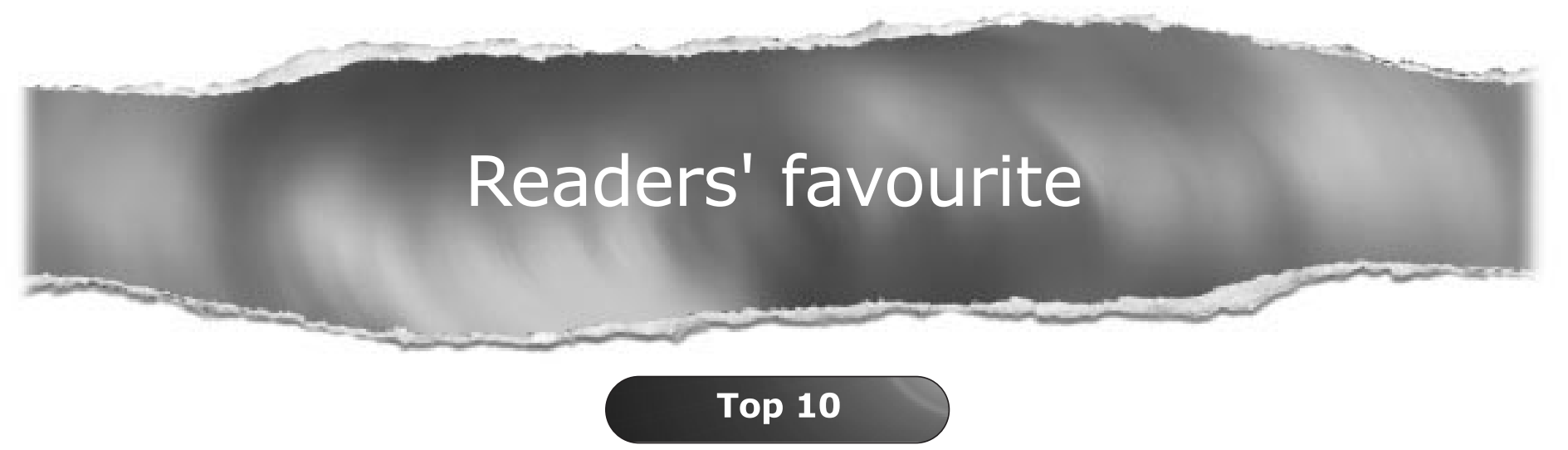

Click on the "Top 10" button on the homepage to see which are the best read articles each month

www.jclinpath.com 